\title{
Cardiovascular Safety of SGLT2 Inhibitors Compared to DPP4 Inhibitors and Sulfonylureas as the Second- Line of Therapy in T2DM Using Large, Real-World Clinical Data in Korea
}

\author{
Kyuho Kim ${ }^{1}$, Sung Hee Choi ${ }^{1,2}$ \\ ${ }^{1}$ Department of Internal Medicine, Seoul National University Bundang Hospital, Seongnam, \\ ${ }^{2}$ Department of Internal Medicine, Seoul National University College of Medicine, Seoul, Korea
}

After approval of the first sodium glucose cotransporter 2 (SGLT2) inhibitors in 2013, SGLT2 inhibitors have been spotlighted due to their cardiovascular and renal benefits according to many randomized controlled trials (RCTs) [1-4]. Most updated guidelines recommended SLGT2 inhibitors as the preferred option for patients with established atherosclerotic cardiovascular disease (ASCVD) or indicators of high ASCVD risk, heart failure (HF), and chronic kidney disease (CKD) $[5,6]$. Based on positive results from the landmark trials (Dapagliflozin and Prevention of Adverse Outcomes in Heart Failure [DAPA-HF] [7] and The Empagliflozin Outcome Trial in Patients with Chronic Heart Failure and a Reduced Ejection Fraction [EMPEROR-Reduced] [8]), dapagliflozin and empagliflozin were approved for reducing the risk of $\mathrm{CV}$ death and hospitalization in patients with heart failure with reduced ejection fraction (HFrEF), both with and without type 2 diabetes mellitus [9]. Still, metformin is the first-line therapy for type 2 diabetes mellitus according to most guidelines, unless there are contraindications. For patients without established ASCVD, indicators of high ASCVD risk, HF, or CKD, the choice of a second-line therapy to add to metformin is not yet prioritized with solid evidence, but rather by considering many clinical profiles of individual patients [6].

Jeon et al. [10] reported the cardiovascular safety of SGLT2 inhibitors compared to dipeptidyl peptidase-4 (DPP-4) inhibitors and sulfonylureas (SU) in a large real-world Korean cohort using National Health Insurance Service data [10]. They showed that SGLT2 inhibitors as a second-line therapy were associated with decreased risk of hospitalization for heart failure (HHF; -22\%) and HHF plus all-cause mortality (-21\%) compared with DPP-4 inhibitors; SGLT2 inhibitors are also associated with decreased risk of HHF (-34\%), all-cause mortality $(-40 \%)$, myocardial infarction (MI; $-35 \%)$, stroke $(-26 \%)$, and modified major adverse cardiovascular events (MACEs; all-cause mortality, MI, and stroke; $-31 \%$ ) compared with SU after the propensity score matching method. The study population included those lower than $20 \%$ of the established cardiovascular disease at baseline; thus, the enrolled subjects with metformin failure in this study for second-line therapy might be at low risk for developing MACE. In other words, they suggested that beneficial effects of SGLT2 inhibitors on cardiovascular composite outcomes might be expected in general populations of patients with type 2 diabetes mellitus.

SGLT2 inhibitors as an add-on to metformin monotherapy showed similar glucose lowering efficacy compared with SU and DPP-4 inhibitors. In a previous head-to-head trial, empagliflozin as an add-on to metformin monotherapy showed non-inferior efficacy at week 52 (relative glycosylated hemo-
Corresponding author: Sung Hee Choi iD https://orcid.org/0000-0003-0740-8116 Department of Internal Medicine, Seoul National University Bundang Hospital, Seoul National University College of Medicine, 82 Gumi-ro 173beon-gil, Bundang-gu, Seongnam 13620, Korea

E-mail: shchoimd@gmail.com
This is an Open Access article distributed under the terms of the Creative Commons Attribution Non-Commercial License (https://creativecommons.org/licenses/by-nc/4.0/) which permits unrestricted non-commercial use, distribution, and reproduction in any medium, provided the original work is properly cited. 
globin [HbA1c] reduction; $-0.07 \%)$, superior efficacy at week 104 (relative $\mathrm{HbAlc}$ reduction; $-0.11 \%$ ), and a low risk of hypoglycemia (2\%) compared with glimepiride (24\%) [11]. Dapagliflozin and canagliflozin showed non-inferior efficacy compared with glipizide and glimepiride, respectively $[12,13]$. Meanwhile, as an add-on to metformin monotherapy, canagliflozin 100 and $300 \mathrm{mg}$ showed non-inferior efficacy, and canagliflozin $300 \mathrm{mg}$ showed superior efficacy compared with sitagliptin (relative HbA1c reduction; -0.15\%) at week 52 [14]. An indirect meta-analysis comparison of dapagliflozin as an add-on to metformin monotherapy demonstrated similar HbAlc control compared with DPP-4 inhibitors after 1 year of treatment [15].

Considering the low risk of hypoglycemia, weight benefits, cardiovascular and renal benefits, and similar glucose-lowering efficacy compared with other oral hypoglycemic agents [16], it is reasonable to give priority to SGLT2 inhibitors as a second-line therapy after metformin monotherapy in patients with type 2 diabetes mellitus in the future, in particular over ASCVD or CKD predominates.

\section{CONFLICTS OF INTEREST}

No potential conflict of interest relevant to this article was reported.

\section{REFERENCES}

1. Zinman B, Wanner C, Lachin JM, Fitchett D, Bluhmki E, Hantel S, et al. Empagliflozin, cardiovascular outcomes, and mortality in type 2 diabetes. N Engl J Med 2015;373:2117-28.

2. Neal B, Perkovic V, Mahaffey KW, de Zeeuw D, Fulcher G, Erondu N, et al. Canagliflozin and cardiovascular and renal events in type 2 diabetes. N Engl J Med 2017;377:644-57.

3. Wiviott SD, Raz I, Bonaca MP, Mosenzon O, Kato ET, Cahn A, et al. Dapagliflozin and cardiovascular outcomes in type 2 diabetes. N Engl J Med 2019;380:347-57.

4. Perkovic V, Jardine MJ, Neal B, Bompoint S, Heerspink HJL, Charytan DM, et al. Canagliflozin and renal outcomes in type 2 diabetes and nephropathy. N Engl J Med 2019;380:2295-306.

5. Kim MK, Ko SH, Kim BY, Kang ES, Noh J, Kim SK, et al. 2019 Clinical practice guidelines for type 2 diabetes mellitus in Korea. Diabetes Metab J 2019;43:398-406.

6. American Diabetes Association. 9. Pharmacologic approaches to glycemic treatment: standards of medical care in diabe- tes-2021. Diabetes Care 2021;44(Suppl 1):S111-24.

7. McMurray JJV, Solomon SD, Inzucchi SE, Kober L, Kosiborod MN, Martinez FA, et al. Dapagliflozin in patients with heart failure and reduced ejection fraction. N Engl J Med 2019;381: 1995-2008.

8. Packer M, Anker SD, Butler J, Filippatos G, Pocock SJ, Carson P, et al. Cardiovascular and renal outcomes with empagliflozin in heart failure. N Engl J Med 2020;383:1413-24.

9. Writing Committee, Maddox TM, Januzzi JL Jr, Allen LA, Breathett K, Butler J, et al. 2021 Update to the 2017 ACC expert consensus decision pathway for optimization of heart failure treatment: answers to 10 pivotal issues about heart failure with reduced ejection fraction: a report of the American College of Cardiology Solution Set Oversight Committee. J Am Coll Cardiol 2021;77:772-810.

10. Jeon JY, Ha KH, Kim DJ. Cardiovascular safety of sodium glucose cotransporter 2 inhibitors as add-on to metformin monotherapy in patients with type 2 diabetes mellitus. Diabetes Metab J 2021;45:505-14.

11. Ridderstrale M, Andersen KR, Zeller C, Kim G, Woerle HJ, Broedl UC, et al. Comparison of empagliflozin and glimepiride as add-on to metformin in patients with type 2 diabetes: a 104week randomised, active-controlled, double-blind, phase 3 trial. Lancet Diabetes Endocrinol 2014;2:691-700.

12. Nauck MA, Del Prato S, Meier JJ, Duran-Garcia S, Rohwedder $\mathrm{K}$, Elze $\mathrm{M}$, et al. Dapagliflozin versus glipizide as add-on therapy in patients with type 2 diabetes who have inadequate glycemic control with metformin: a randomized, 52-week, doubleblind, active-controlled noninferiority trial. Diabetes Care 2011; 34:2015-22.

13. Cefalu WT, Leiter LA, Yoon KH, Arias P, Niskanen L, Xie J, et al. Efficacy and safety of canagliflozin versus glimepiride in patients with type 2 diabetes inadequately controlled with metformin (CANTATA-SU): 52 week results from a randomised, double-blind, phase 3 non-inferiority trial. Lancet 2013;382: 941-50.

14. Lavalle-Gonzalez FJ, Januszewicz A, Davidson J, Tong C, Qiu R, Canovatchel W, et al. Efficacy and safety of canagliflozin compared with placebo and sitagliptin in patients with type 2 diabetes on background metformin monotherapy: a randomised trial. Diabetologia 2013;56:2582-92.

15. Goring S, Hawkins N, Wygant G, Roudaut M, Townsend R, Wood I, et al. Dapagliflozin compared with other oral anti-diabetes treatments when added to metformin monotherapy: a systematic review and network meta-analysis. Diabetes Obes 
Metab 2014;16:433-42.

16. Avogaro A, Delgado E, Lingvay I. When metformin is not enough: pros and cons of SGLT2 and DPP-4 inhibitors as a second line therapy. Diabetes Metab Res Rev 2018;34:e2981. 